\title{
Inherited heart rhythm disorders: Diagnostic dilemmas after the sudden death of a young family member
}

\author{
Mikyla L J anzen ${ }^{1}$, Shubhayan Sanatani ${ }^{2}$, Karen A Gibbs ${ }^{1}$, Saira S Mohammed ${ }^{2}$, J ulie Hathaway ${ }^{1}$, \\ Laura Arbour ${ }^{3}$, Andrew D Krahn ${ }^{1}$ \\ 1. Division of Cardiology, University of British Columbia, Vancouver, Canada. 2. BC Children's Hospital, Vancouver, \\ Canada. 3. Department of Medical Genetics, University of British Columbia, Island Medical Program, Victoria, British \\ Columbia, Canada.
}

Correspondence: Andrew Krahn. Address: ${ }^{\text {th }}$ Floor, Gordon \& Leslie Diamond Health Care Centre, 2775 Laurel Street, Vancouver, BC V5Z 1M9, Canada. Email: akrahn@mail.ubc.ca

Received: July 31, 2013

Accepted: September 4, 2013

Online Published: January 12, 2014

DOI : $10.5430 /$ jnep.v4n3p225

URL: http://dx.doi.org/10.5430/jnep.v4n3p225

\begin{abstract}
Inherited heart rhythm disorders (IHRD) are uncommon conditions that are often not detected until the tragic sudden unexpected death (SUD) of a seemingly healthy young individual. These conditions include Long QT Syndrome (LQTS), Arrhythmogenic Right Ventricular Cardiomyopathy (ARVC), Catecholaminergic Polymorphic Ventricular Tachycardia (CPVT), and Brugada Syndrome. Due to their rarity and wide variety of presentations, IHRD are often not suspected and, even when they are considered, can be difficult to diagnose. As a result, many people are left undiagnosed, with a potential “time bomb” ticking with every heartbeat. In early 2013, the British Columbia Inherited Arrhythmia Program (BC IAP) was launched by a team of adult and pediatric electrophysiologists, medical geneticists, genetic counselors, clinical and research nurses, and other health professionals. Unfortunately, warning signs of IHRD may be subtle, missed or nonexistent, and thus cardiac arrest or SUD is often the reason for referral to the BC IAP. In an effort to increase awareness and prevent SUD, the BC IAP provides clinical consultation and genetic counseling to index cases (probands) and their families. The comprehensive, multidisciplinary program specializes in the diagnosis, management, and innovative research of IHRD. Here, the proband and their family are supported through the process of diagnostic testing that screens each first degree family member and helps find answers, reveals potential inherited arrhythmia conditions, and potentially plays a crucial role in future research of these rare and incompletely defined conditions.
\end{abstract}

\section{Key words}

Diagnosis, Genetics, Sudden death

\section{Brief summary}

Although uncommon, inherited heart rhythm disorders (IHRD) can result in sudden death in apparently healthy young people and prove to be a difficult diagnosis for physicians to make. The British Columbia Inherited Arrhythmia Program (BC IAP) combines experts in cardiac electrophysiology and genetics to determine the correct diagnosis and treatment, while providing support and appropriate screening for families affected by these conditions. 


\section{Objectives}

The purpose of this review is to:

1) Outline the clinical presentation of the inherited heart rhythm disorders (IHRDs), and the use of clinical history including family history, along with diagnostic testing to detect IHRDs

2) Briefly discuss the individual underlying conditions, with a focus on specific diagnostic and therapeutic considerations

3) Outline the process of formulation of a regional expert program in IHRD recognition and management

\section{Background}

IHRD are a group of relatively uncommon conditions that lead to familial fainting, cardiac arrest and sudden death. Many physicians are unfamiliar with the IHRDs due to their relative rarity, a lack of emphasis in medical education, and a lack of general medical awareness ${ }^{[1]}$. However, these familial conditions can result in sudden unexplained death (SUD) in seemingly healthy children or young adults. This group of conditions includes primary electrical diseases that predominantly affect cardiac repolarization, and uncommon "mechanical" diseases that affect cardiac muscle. In British Columbia alone, it is estimated that 7,000 individuals are at risk and up to 45 will suddenly die each year due to IHRD. Most importantly, the majority of these deaths can be effectively and inexpensively prevented if the underlying condition is diagnosed. However, the varying presentations and relative scarcity of such disorders can serve as a diagnostic challenge and thus a correct and definitive diagnosis can be difficult. It is critical to identify and appropriately manage these disorders; if left unrecognized and untreated, arrhythmias can lead to cardiac arrest and SUD.

Although young adults often believe they are invincible to the world's harm, IHRD contradict this notion. In fact, these conditions are most common in adolescents and young adults. Whether this predilection is due to developmental, physical, or other factors is incompletely understood. Nevertheless, the false perception of invincibility can change in the blink of an eye. Many arrhythmogenic events occur without warning, ranging from syncope (fainting) to sudden death.

\section{Diagnostic considerations and issues}

IHRD are relatively new to the clinical scene; though they have been around for decades, much of what we know has been discovered in the last 30 years. Most physicians are not familiar with the subtle diagnostic signs on the patient's electrocardiogram (ECG), and often attribute potential warning symptoms (fainting, dizziness) to more common ailments such as vasovagal syncope (the common faint), epilepsy, or puberty. Specifically, most physicians, including many cardiologists, have difficulty accurately assessing the QT interval, hence a sudden collapse caused by Long QT Syndrome (LQTS) may be misdiagnosed as epilepsy in children ${ }^{[2]}$. Both conditions can present as episodic loss of consciousness, sometimes triggered by sudden, startling noises; epilepsy is more than ten times as prevalent as LQTS ${ }^{[3,4]}$. However, with proper and informed consultation, elements such as a family history of sudden death, bystander history of collapse followed by apparent seizure activity and potentially concerning ECG findings can distinguish LQTS from epilepsy.

\section{The Electrocardiogram (ECG)}

The ECG is a cardiologist's simplest and most accessible tool to visualize alterations in the heart's electrical system suggestive of a specific IHRD. Each category of IHRD can display slightly different abnormalities in the heart's electrical activity, as described in Table $1^{[3-5]}$. LQTS is most commonly characterized by a prolonged QT interval caused by an abnormal decrease in potassium channel function or rarely, an increase in sodium channel function. This categorizes LQTS as an intracardiac ion "channelopathy" as the sodium and potassium currents responsible for the cardiac action potential captured on the electrocardiogram (ECG) are abnormal. The resulting loss of function of potassium efflux or gain 
of function of sodium influx leads to prolonged repolarization of the ventricles. Consequently, the heart's electrical balance takes longer to reset, potentially resulting in spontaneous early after depolarizations, leading to the hallmark arrhythmia torsade de pointes, a type of ventricular tachycardia.

Table 1. A summary of the electrical abnormalities and biochemical causes found in inherited arrhythmias

\begin{tabular}{|c|c|c|c|}
\hline $\begin{array}{l}\text { Inherited } \\
\text { Arrhythmia }\end{array}$ & Electrical Abnormality & Cellular Mechanism & Circumstances of Symptoms \\
\hline LQTS & Prolonged repolarization (QT interval) & $\begin{array}{l}\text { Loss of } \mathrm{K}^{+} \text {channel function } \\
\text { Gain of } \mathrm{Na}^{+} \text {channel function }\end{array}$ & $\begin{array}{l}\text { Sudden noises, exercise, } \\
\text { swimming, QT prolonging drugs }\end{array}$ \\
\hline ARVC & $\begin{array}{l}\text { Inverted } \mathrm{T} \text { waves in the anterior } \\
\text { precordial leads } \\
\text { Prolonged depolarization } \\
\text { epsilon waves }\end{array}$ & $\begin{array}{l}\text { Loss of integrity of cardiac } \\
\text { desmosomes }\end{array}$ & $\begin{array}{l}\text { No typical trigger, occasionally } \\
\text { exercise }\end{array}$ \\
\hline CPVT & $\begin{array}{l}\text { Irregular polymorphic ventricular } \\
\text { tachycardia }\end{array}$ & $\begin{array}{l}\mathrm{Ca}^{++} \text {overload inside the } \\
\text { cardiac cell }\end{array}$ & Exercise or stress \\
\hline Brugada & $\begin{array}{l}\text { ST elevation in the anterior precordial } \\
\text { leads, especially with high precordial } \\
\text { leads }\end{array}$ & $\mathrm{Na}^{+}$channelopathy & Rest or sleep \\
\hline
\end{tabular}

Arrhythmogenic Right Ventricular Cardiomyopathy (ARVC) mainly affects the right ventricle and is associated with several ECG abnormalities, including inverted T waves and prolonged depolarization, as well as structural changes in the cardiac muscle. ARVC disrupts the cardiac cell connections (called desmosomes), part of the complex apparatus that holds the heart cells together to ensure proper propagation of the electrical signal and contractility. The clinical events associated with this disorder may occur during exercise, particularly in young males ${ }^{[6]}$.

Due to their catecholaminergic nature, Catecholaminergic Polymorphic Ventricular Tachycardia (CPVT) provoked arrhythmias usually require exercise or emotional stress to be induced and cause a rapid ventricular arrhythmia, which can result in SUD. The majority of Catecholaminergic Polymorphic Ventricular Tachycardia (CPVT) cases are caused by abnormalities in calcium handling, also categorizing this disorder as an ion channelopathy. Around $60 \%$ of CPVT is due to mutations in the transmembrane ryanodine receptor which serves as a calcium channel; this large and complex structure is encoded by the RyR2 gene.

Finally, Brugada syndrome is a disorder of failed depolarization, most often attributed to reduced sodium current ${ }^{[5]}$. Recent evidence suggests that it is considerably more complex than this simple loss of function explanation. The ECG finding alone is called Brugada pattern, and when combined with symptoms is called Brugada Syndrome. There are three types of Brugada patterns: the type 1 pattern, whether spontaneous or provoked by a sodium channel blocker, is the only one of clinical concern. Type 2 is the most common and Type 3 has not been shown to significantly increase cardiac risk. Sometimes, changing the position of the precordial ECG leads to 1 or 2 intercostal spaces higher is required to detect one of these patterns. Brugada syndrome is more common in males and Asians, usually manifesting as syncope, seizures or SUD during sleep or rest in young males.

The diagnosis of IHRDs is often straightforward in overtly affected individuals, but much more challenging in the majority of cases because of variable penetrance (the extent to which individuals with the same genotype display the same phenotype) and expressivity (variations in the phenotype with the same or similar mutations). In simple terms, rare but dramatic arrhythmias occur in an individual as a "perfect storm" when the coincidence of genetic susceptibility, environmental factors and a trigger such as exercise or a loud noise lead to life-threatening arrhythmia. Outside of this immediate period, the patient often has much less compelling evidence of disease, making a clear diagnostic signature less than obvious. Thus the majority of the IHRDs are approached with a broad spectrum of testing to detect clinical and 
genetic evidence of disease, or provocation is conducted to unmask the diagnosis with maneuvers such as exercise testing or cardiac monitoring.

Although it is currently the simplest and most accessible diagnostic test, the ECG is not always an ideal screening tool. Not all genetically affected individuals harbor clinical abnormalities (phenotype), and some cases show only sporadic diagnostic changes on an ECG. Because symptoms can vary with time, a range of ECGs is often useful to detect abnormalities that may be transient ${ }^{[1]}$. A resting ECG creates a snapshot of the heart's electrical activity; low-voltage abnormalities are often corrected and attributed to the normal variance of the heart. A signal averaged ECG (signal averaged ECG) combines electrical data from around 400 heartbeats: this technique can reveal small abnormalities in the electrical signal that would be overlooked on a normal ECG ${ }^{[7,8]}$. The most prominent diagnostic issue arises in patients who do not harbor symptoms or a history of cardiac events, and have subtle or transient ECG abnormalities. Thus, the importance of proper awareness and education of the clinical staff is imperative. Additional testing that is often considered in patients at risk includes exercise testing, ambulatory monitoring, cardiac ultrasound (echocardiography), cardiac MRI and drug challenge (procainamide or epinephrine infusion) ${ }^{[8]}$.

\section{Genetic considerations}

Most IHRD are inherited in an autosomal manner, caused by mutations, or "typos", in specific areas of the DNA code (genes). A new mutation can occur in any generation, and can be passed from parent to child. IHRD are typically transmitted in an autosomal dominant fashion, meaning only one copy of an altered or "mutated" gene is required to result in the condition predisposing to cardiac arrest or sudden death. Therefore, an affected individual has a 50\% chance of passing on the genetic predisposition to the specific IHRD to each of his or her children. However, of two family members with an identical genetic mutation, one may present a phenotype including symptoms while another may be asymptomatic with much less evidence that they are affected ${ }^{[9,10]}$. This is due to phenomena called reduced penetrance and variable expressivity. Reduced penetrance means that not every patient with a known pathogenic mutation shows features of the disease. Variable expressivity refers to the wide range and combinations of sign and symptoms seen in patients with IHRD.

With recent genetic sequencing advances and the familial nature of these conditions, genetic testing commonly plays a large role in the diagnosis of IHRD. As tempting as it can be to genetically test every potential IHRD patient, there are still a number of drawbacks to be considered. Besides the substantial financial limitation (testing costs around $\$ 3000$ for the proband), there are social, psychological, and scientific considerations. Social concerns stem from insurance and employment discrimination; the tested individual may be unable to obtain life insurance or pursue certain careers. This is also true for clinical cardiac testing, and asymptomatic family members should be aware of this potential before undergoing genetic screening. Compounded with the variable penetrance and expressivity of these conditions, asymptomatic mutation carriers may be unnecessarily discriminated against. The alternative perspective includes confirmation one does not carry the family mutation, which may have a positive effect on insurance eligibility. The psychosocial burden of living with a gene mutation predisposing to sudden death also needs to be considered before genetic testing. Families are commonly referred only after a tragic death, and although there is no control over which copy of a gene is passed on to a child, a parent may feel immense guilt if found to have the mutation identified in the deceased. Finally, there is also a substantial scientific limitation as only a modest proportion of patients will harbor a disease-causing mutation (5-75\% based on underlying condition) ${ }^{[11-13]}$. In addition, a genetic test may reveal a disease-causing mutation, a variant of unknown significance (VUS) (a "maybe” mutation), or no known mutation. These VUS may represent a minor difference in one's genes leading to individuality; however, they could also be the explanation for an IHRD. Patients, particularly those undergoing predictive or presymptomatic testing, should consider whether they wish to accept awareness of what lies within their genes before delving into genetic testing. However, unlike many other genetic disease models for presymptomatic testing where no treatment is available ${ }^{[14]}$, the recognition of a 'predisposition to' an IHRD may allow for preventative treatment, such as avoidance of QT prolonging drugs and the use of beta blockers in LQTS1 ${ }^{[15]}$. 


\section{The BC I nherited Arrhythmia Program}

Since its inception in early 2013, the BC IAP's mission has been to provide state-of-the-art care for families affected by heart rhythm conditions through partnerships, innovation, and advocacy. Often a tragedy is the catalyst for referral to the BC IAP. The sudden unexpected death of a loved one can subject the bereaved to an overwhelming reaction of shock and grief, regardless of the circumstance. In the case of IHRD, this loved one is typically young and seemingly healthy, making the loss more difficult for the entire family. Due to the familial nature of these conditions, the BC IAP commonly assesses patients through a family-based approach, naturally creating a support system for each person within the family. Once referred, the comprehensive BC IAP team can support families making decisions around genetic testing, diagnostics, and treatment options (see Figure 1).

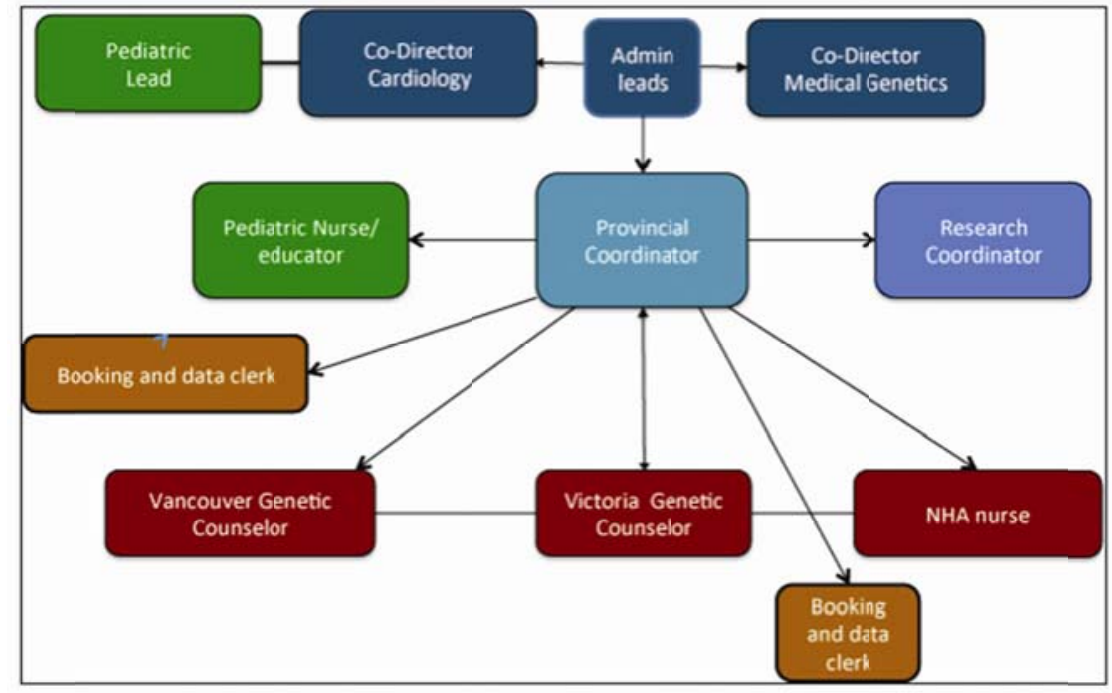

Figure 1. Organizational chart for the BC Inherited Arrhythmia Program team.

In the event of a sudden unexplained cardiac arrest or a SUD, it is important for both the proband and first-degree relatives to be evaluated. First-degree relatives should also be tested for potential warning signs that may have been undetected in the deceased following sudden cardiac death. This process of assessing first-degree relatives of clinically and/or genetically affected individuals is called cascade screening. Hence, a holistic familial approach must be taken to ensure the safety and protection from SUD for all family members ${ }^{[16]}$.

Once referred, a comprehensive personal and family history is discussed with each new patient to help focus the investigation ${ }^{[12,17]}$. The personal history can reveal potential warning signs that may be relevant to a potential IHRD, such as syncope, crib deaths (SIDS) or frequent miscarriages. A thorough family history is vital to bring together the varying presentations of these conditions and gain guiding knowledge of previous family members' cardiac events and/or deaths. For example, a patient with an aunt who died in a single motor vehicle accident, and a stillborn sibling may never piece together that a familial condition may tie these events together. This information is then combined with the results of a resting ECG, exercise test, ambulatory monitor and/or SAECG to find electrical clues, and autopsy details (if any) to create a working diagnosis. Once a preliminary diagnosis is established, further specific tests can be administered to attempt to confirm the diagnosis (see Figure 2). Exercise tests and epinephrine infusion challenges can serve to potentiate prolonged QT segments and confirm LQTS. Structural abnormalities can be investigated with an echocardiogram or MRI, useful to diagnose ARVC and other cardiomyopathies, but are usually normal in ion channel-pathies. Provocative testing with procainamide can unmask the Brugada pattern and thus assist with the determination of the risk and necessary treatment (see Figure 3). 


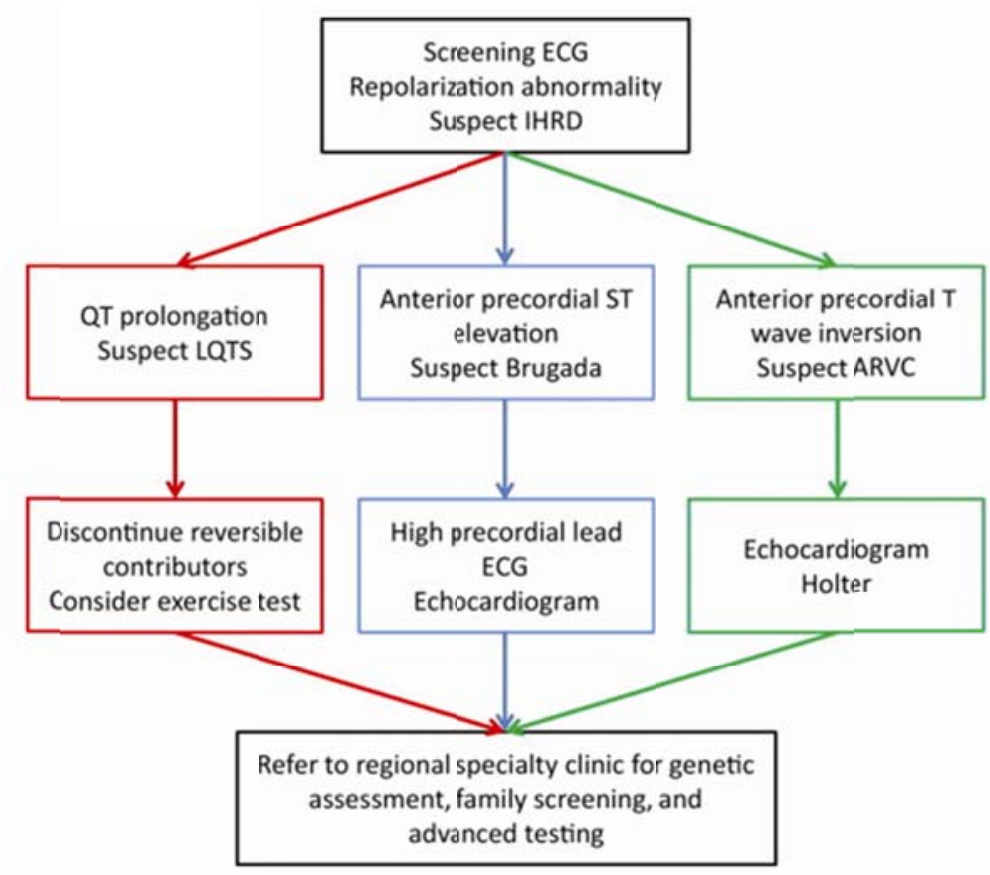

Figure 2. Initial clinical management scheme for Inherited Heart Rhythm Disorders (IHRD) ${ }^{[5]}$

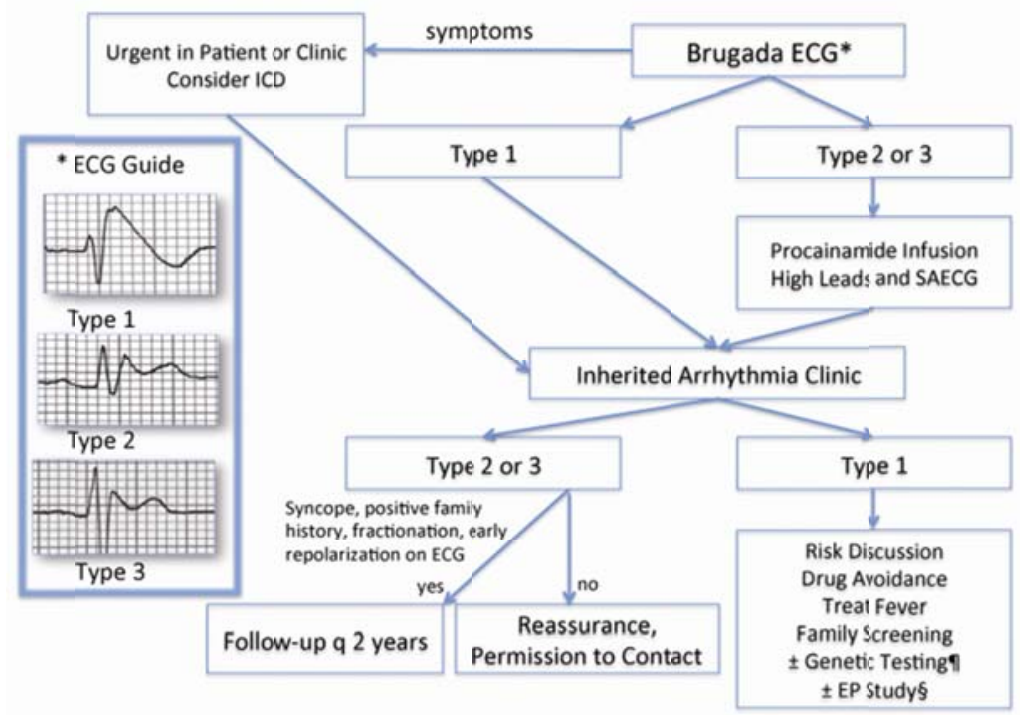

Figure 3. An overview of Brugada Syndrome diagnosis and care

Another common hurdle challenge in the assessment of families after the SUD of their loved one regards the decedent's autopsy. In most jurisdictions, an autopsy is either mandatory or strongly recommended if death is unexplained ${ }^{[13]}$. Preliminary results may or may not be communicated to the family and final results are typically not available for several months. For those with IHRDs, the autopsy typically has no identified cause of death, and subtle evidence of structural abnormalities that suggest ARVC may require the expertise of a cardiac pathologist to correctly diagnose. Since the heart's electrical system ceases to function with death, there can be no forensic inspection of the electrical system to speak of, so genetic tools are needed to provide insight. If a disease causing mutation is found (25\%-40\% of cases), direct testing can be offered to family members. As ideal and simple as this situation sounds, unfortunately it is not yet the standard process. Even if a disease causing mutation is potentially present, post mortem samples are not routinely secured and adequate 
DNA may not be available for testing following the autopsy. Furthermore, given the patient is deceased, it is often unclear as to whose insurance should pay the costly bill for testing. Given universal health care in Canada, and the substantial effect on living members of the family, the BC IAP is committed to increasing awareness around the clinical utility of preserving such samples, and pursing testing to confirm a disease causing mutation for those family members who remain at risk.

Detailed discussion of the therapies for IHRD is beyond the scope of this review. Once an initial diagnosis is arrived at, treatment options are discussed with families. Lifestyle advice including exercise advice, drugs to avoid and reporting of symptoms are universal education points. Generally, patients diagnosed with adrenergically mediated conditions (CPVT or LQTS) are given daily doses of beta-blockers which act to prevent sudden changes in heart rate. These drugs prevent abrupt heart rate acceleration, and thus prophylactically prevent life-threatening arrhythmias. However, beta-blockers are a treatment, not a cure; drug non-compliance is one of the recognized contributing factors in that perfect storm ${ }^{[15]}$. Cardiac arrest survivors are generally offered an implantable cardioverter defibrillator (ICD). This device provides life-saving defibrillation when ventricular arrhythmias occur, but do have downfalls. The implantation is an invasive surgery, ICDs can produce inappropriate shocks, and currently, need to be replaced every 6-10 years.

Depending on the diagnosis, certain prescription and over-the-counter drugs, co-morbidities, and activities should also be avoided; extensive lists are available online at www.brugadadrugs.org and www.qtdrugs.org. Brugada symptoms can be exacerbated by fever, so all patients are encouraged to aggressively treat fever with acetaminophen. Exercise recommenddations are controversial but important points for personal and family discussions - the physical and mental benefits of exercise are known, but it may exacerbate the effects of conditions such as ARVC, LQTS and CPVT. Fortunately, the BC IAP can provide guidance, support, and care for families diagnosed with inherited arrhythmias who have questions regarding sports and physical activity.

\section{Research at the BC I nherited Arrhythmia Program}

Research drives improvements in health care. IHRD are complex and many questions remain about the diagnosis and management. These conditions have historically been the template for "bench to bedside" research: What causes two family members with the same genotype (specific DNA sequence) to have at times dramatically different phenotypes (clinical presentation of the genetic condition)? Beyond simple single gene explanations, how do our many other genetic contributors influence risk for patients? Like so many paroxysmal diseases, what are the temporal factors that make the "lightning strike" when it does? What are the best techniques for enhancing family communication of important and potentially life-threatening information? What is the best method for educating health care professionals to enhance awareness and thus preventive efforts in IHRDs? Are there more categories of conditions that are currently being misdiagnosed? Are there certain symptoms that infer a greater predisposition to cardiac arrest and death than others? Currently, only a few mutations have confirmed functional data to help us understand their effect on the heart's electrical system, limiting genetic testing's sensitivity and accuracy. Beta-blockers are typically effective as outlined above, but there is no established beneficial drug therapy for ARVC or Brugada Syndrome. Modern treatment measures can also fail as patients may be unresponsive to beta-blockers and complications can arise from ICDs. These limitations of care drive research initiatives to discover improved diagnosis and therapy for IHRDs, and better understand the genetic contributors.

The British Columbia Inherited Arrhythmia Research Team is an interdisciplinary group that includes clinical staff and patient/family advisory representation. Currently, each patient seen at the BC IAP is invited to participate in the BC Inherited Arrhythmia Research Registry and Bio Bank and provide permission to contact for future research. The BC Inherited Arrhythmia Research Registry and Bio Bank is an ongoing confidential provincial database of de-identified demographic and clinical information paired with frozen plasma, RNA and DNA. The physician team of the BC IAP (Drs. Krahn, Sanatani and Arbour) and the University of British Columbia Inherited Arrhythmia Research Group lead provincial and national registries and bio banks: CASPER (Cardiac Arrest Survivors with Preserved Ejection Fraction Registry) and the Canadian National Arrhythmogenic Right Ventricular Cardiomyopathy (ARVC) Registry ${ }^{[14]}$. Firstly, 
the CASPER database and bio bank includes data of cardiac arrest survivors and their family members across Canada. Since 2005, this registry has been collecting data on patients who lack a known cause of their own or their family member's cardiac arrest or sudden death. Secondly, the Canadian National ARVC Registry and Bio Bank is the first of several planned condition-specific registries. This database gathers data paired with biobanked specimens from Canadians with confirmed or suggested ARVC and their first-degree family members. A proposed Canadian National Long QT Syndrome Registry and Bio Bank will provide much needed data and bio specimens to drive research specific for those affected with LQTS.

All of these registries are imperative tools for future research as they pool patients with rare conditions, allowing large enough sample sizes for data analysis and the outcome of significant results with the potential impact to improve patient care and reduce mortality. Furthermore, the large collection of data from these registries provides a diverse spectrum of probands (index case that is first identified in a family) and first-degree family members with varying circumstances. This allows for a more thorough understanding of the etiology and characterization, both genetic and clinical, of IHRD. The common goal is to create standardized diagnostic and treatment procedures for inherited arrhythmias and prevent unnecessary sudden deaths in Canada.

\section{Acknowledgements}

The BCIAP was funded originally through the BC Ministry of Health, British Columbia Medical Association joint Specialty Services Committee competition for innovation in health services delivery. The ongoing funding is supported by Cardiac Services BC.

\section{References}

[1] Huisma FF, Potts JE, Gibbs KA, Sanatani S. Assessing the knowledge of sudden unexpected death in the young among canadian medical students and recent graduates: A cross-sectional study. BMJ Open. 2012;2

[2] Viskin S, Rosovski U, Sands AJ, Chen E, Kistler PM, Kalman JM, Rodriguez Chavez L, Iturralde Torres P, Cruz FF, Centurion OA, Fujiki A, Maury P, Chen X, Krahn AD, Roithinger F, Zhang L, Vincent GM, Zeltser D. Inaccurate electrocardiographic interpretation of long qt: The majority of physicians cannot recognize a long qt when they see one. Heart Rhythm. 2005; 2: 569-574. PMid:15922261 http://dx.doi.org/10.1016/j.hrthm.2005.02.011

[3] Ngugi AK, Bottomley C, Kleinschmidt I, Sander JW, Newton CR. Estimation of the burden of active and life-time epilepsy: A meta-analytic approach. Epilepsia. 2010; 51: 883-890. PMid:20067507 http://dx.doi.org/10.1111/j.1528-1167.2009.02481.x

[4] Jackson H, Huisman LA, Sanatani S, Arbour LT. Long qt syndrome. CMAJ. 2011; 183: 1272-1275. PMid:21482651 http://dx.doi.org/10.1503/cmaj.100138

[5] Krahn AD, Sanatani S, Gardner MJ, Arbour L. Inherited heart rhythm disease: Negotiating the minefield for the practicing cardiologist. Can J Cardiol. 2013;29:122-125. PMid:23261322 http://dx.doi.org/10.1016/j.cjca.2012.11.009

[6] Romero J, Mejia-Lopez E, Manrique C, Lucariello R. Arrhythmogenic right ventricular cardiomyopathy (arvc/d): A systematic literature review. Clin Med Insights Cardiol. 2013; 7: 97-114. PMid:23761986

[7] Kamath GS, Zareba W, Delaney J, Koneru JN, McKenna W, Gear K, Polonsky S, Sherrill D, Bluemke D, Marcus F, Steinberg JS. Value of the signal-averaged electrocardiogram in arrhythmogenic right ventricular cardiomyopathy/dysplasia. Heart Rhythm. 2011; 8: 256-262. PMid:20933608 http://dx.doi.org/10.1016/j.hrthm.2010.10.007

[8] Krahn AD, Healey JS, Chauhan V, Birnie DH, Simpson CS, Champagne J, Gardner M, Sanatani S, Exner DV, Klein GJ, Yee R, Skanes AC, Gula LJ, Gollob MH. Systematic assessment of patients with unexplained cardiac arrest: Cardiac arrest survivors with preserved ejection fraction registry (casper). Circulation. 2009; 120: 278-285. PMid:19597050

http://dx.doi.org/10.1161/CIRCULATIONAHA.109.853143

[9] Priori SG, Napolitano C, Schwartz PJ. Low penetrance in the long-qt syndrome: Clinical impact. Circulation. 1999 Feb 2; 99 : 529-533. PMid:9927399 http://dx.doi.org/10.1161/01.CIR.99.4.529

[10] Amin AS, Giudicessi JR, Tijsen AJ, Spanjaart AM, Reckman YJ, Klemens CA, Tanck MW, Kapplinger JD, Hofman N, Sinner MF, Muller M, Wijnen WJ, Tan HL, Bezzina CR, Creemers EE, Wilde AA, Ackerman MJ, Pinto YM. Variants in the 3' 
untranslated region of the kcnq1-encoded kv7.1 potassium channel modify disease severity in patients with type 1 long qt syndrome in an allele-specific manner. Eur Heart J. 2012; 33: 714-723. PMid:22199116 http://dx.doi.org/10.1093/eurheartj/ehr473

[11] Bennett MT, Sanatani S, Chakrabarti S, Deyell MW, Krahn AD. Assessment of genetic causes of cardiac arrest. Can J Cardiol. 2013; 29: 100-110. PMid:23200097 http://dx.doi.org/10.1016/j.cjca.2012.07.002

[12] Gollob MH, Blier L, Brugada R, Champagne J, Chauhan V, Connors S, Gardner M, Green MS, Gow R, Hamilton R, Harris L, Healey JS, Hodgkinson K, Honeywell C, Kantoch M, Kirsh J, Krahn A, Mullen M, Parkash R, Redfearn D, Rutberg J, Sanatani S, Woo A. Recommendations for the use of genetic testing in the clinical evaluation of inherited cardiac arrhythmias associated with sudden cardiac death: Canadian cardiovascular society/canadian heart rhythm society joint position paper. Can J Cardiol. 2011; 27: 232-245. PMid:21459272 http://dx.doi.org/10.1016/j.cjca.2010.12.078

[13] Ackerman MJ, Priori SG, Willems S, Berul C, Brugada R, Calkins H, Camm AJ, Ellinor PT, Gollob M, Hamilton R, Hershberger RE, Judge DP, Le Marec H, McKenna WJ, Schulze-Bahr E, Semsarian C, Towbin JA, Watkins H, Wilde A, Wolpert C, Zipes DP. Hrs/ehra expert consensus statement on the state of genetic testing for the channelopathies and cardiomyopathies: This document was developed as a partnership between the heart rhythm society (hrs) and the european heart rhythm association (ehra). Europace. 2011; 13: 1077-1109. PMid:21810866 http://dx.doi.org/10.1093/europace/eur245

[14] Agostinho LA, Dos Santos SR, Alvarenga RM, Paiva CL. A systematic review of the intergenerational aspects and the diverse genetic profiles of huntington's disease. Genet Mol Res. 2013; 12: 1974-1981. PMid:23913380 http://dx.doi.org/10.4238/2013.June.13.6

[15] Vincent GM, Schwartz PJ, Denjoy I, Swan H, Bithell C, Spazzolini C, Crotti L, Piippo K, Lupoglazoff JM, Villain E, Priori SG, Napolitano C, Zhang L. High efficacy of beta-blockers in long-qt syndrome type 1: Contribution of noncompliance and qt-prolonging drugs to the occurrence of beta-blocker treatment "failures". Circulation. 2009; 119: 215-221. PMid:19118258 http://dx.doi.org/10.1161/CIRCULATIONAHA.108.772533

[16] Ruiter JS, Berkenbosch-Nieuwhof K, van den Berg MP, van Dijk R, Middel B, van Tintelen JP. The importance of the family history in caring for families with long qt syndrome and dilated cardiomyopathy. Am J Med Genet A. 2010; 152A: 607-612. PMid:20186837 http://dx.doi.org/10.1002/ajmg.a.33270

[17] Link MS, Exner DV, Anderson M, Ackerman M, Al-Ahmad A, Knight BP, Markowitz SM, Kaufman ES, Haines D, Asirvatham SJ, Callans DJ, Mounsey JP, Bogun F, Narayan SM, Krahn AD, Mittal S, Singh J, Fisher JD, Chugh SS. Hrs policy statement: Clinical cardiac electrophysiology fellowship curriculum: Update 2011. Heart Rhythm. 2011; 8: 1340-1356. PMid:21699868 http://dx.doi.org/10.1016/j.hrthm.2011.06.008 